\title{
ANÁlisis de la SENSIBILIDAd DE BIOTIPOS DE Lolium multiflorum A HeRbicidas INHIBIDORES DE LA ENZIMA ALS, ACCASA y Glifosato ${ }^{1}$
}

\author{
Sensitivity Analysis of Lolium multiflorum Biotypes to Glyphosate, ACCase and ALS-Inhibiting \\ Herbicides
}

DIEZ DE ULZURRUN, P. ${ }^{2}$ y LEADEN, M.I. ${ }^{3}$

\begin{abstract}
RESUMEN - A pesar de los avances logrados en el control de las malezas con el uso de herbicidas, el manejo de las mismas no se simplificó, sino que, al contrario, surgieron nuevos desafios, como la aparición de resistencia a herbicidas. En 2007, se reportó en Lolium multiflorum el segundo caso de resistencia a glifosato detectado en Argentina. En el sudeste de la provincia de Buenos Aires se registraron fallas de control a campo en poblaciones de Lolium multiflorum debido a su resistencia a distintos herbicidas de las familias de los inhibidores de ALS y de ACCasa y al herbicida glifosato. El objetivo de este estudio fue caracterizar el nivel de resistencia a ciertos herbicidas inhibidores de la ALS y de la ACCasa y al glifosato en una población de L. multiflorum de Lobería (Bs As, Argentina) supuestamente resistente (LmR). Se realizaron bioensayos en cajas de Petri y se determinó la $\mathrm{GR}_{50}$ mediante la variación en la longitud de coleoptile. Las curvas de dosis-respuesta se obtuvieron por medio de la ecuación log-logística. El biotipo LmR presentó resistencia múltiple a herbicidas con tres modos de acción diferentes: glifosato, inhibidores de ALS y de ACCasa. Dicho ensayo demostró la aparición de un biotipo de L. multiflorum con resistencia a múltiples principios activos.
\end{abstract}

Palabras clave: raigrás, resistencia múltiple, bioensayos, sudeste bonaerense.

ABSTRACT - Despite progress in weed control using herbicides, management has not been simplified, but new challenges such as herbicides resistance have arisen. In 2007, a Lolium multiflorum population resistant to glyphosate was reported, as the second case of glyphosate resistant weeds in Argentina. In the southeast of Buenos Aires province, control failures in populations of L. multiflorum to different families of herbicide such as ALS and ACCase inhibitors and to glyphosate at field level have been registered. The aim of this study was to characterize the level of resistance to certain herbicides inhibitors of ALS, ACCase and glyphosate in a putatively resistant (LmR) population of L. multiflorum of Loberia (Buenos Aires, Argentina). Bioassays were conducted in Petri dishes and the $G R_{50}$ was determined by the length of coleoptile. Curves dose-response were obtained using the log-logistic equation. The LmR biotype survived under multiple herbicides of three different modes of action, glyphosate, ALS and ACCase inhibitors. This test showed the appearance of a biotype of $\boldsymbol{L}$. multiflorum with resistance to multiple active ingredients.

Keywords: ryegrass, multiple resistant, bioassay, Southeast of Buenos Aires Province.

\section{INTRODUCCIÓN}

E1 uso generalizado de herbicidas en la agricultura moderna ha incrementado notablemente la producción de alimentos a escala mundial (Powles y Yu, 2010). A pesar de los grandes avances logrados con dicha práctica de control de plagas, la problemática de las malezas no mejoró, sino que, al contrario, surgieron nuevos desafios en su

1 Recebido para publicação em 16.4.2011 e aprovado em 25.6.2012.

2 Becaria de la Universidad Nacional de Mar del Plata, < pdiezulzurrun@balcarce.inta.gov.ar>; ${ }^{3}$ Profesora Adjunta de la Facultad de Ciencias Agrarias de la Universidad Nacional de Mar del Plata, Ruta 226 Km 73,5 Balcarce (Pcia de Buenos Aires), Argentina. 
manejo, como la dominancia de las malezas tolerantes y la aparición de resistencia a los herbicidas de uso predominante.

La Weed Science Society of America (WSSA, 1998) define la resistencia a herbicidas como la habilidad hereditaria que algunos biotipos dentro de una población adquieren para sobrevivir y reproducirse a determinada dosis de un herbicida, a la cual la población original era susceptible. Se asume que cualquier población de malezas puede contener biotipos resistentes en baja frecuencia y que el uso repetido de un mismo herbicida expone la población a una presión de selección que conduce a un aumento en el número de individuos resistentes (Gressel y Segel, 1978).

La dinámica y el impacto de la resistencia depende de varios factores, como la especificidad del herbicida, la diversidad de los genes de resistencia involucrados, y de factores de manejo como la dosis y la frecuencia de uso, entre otros, que van a afectar, principalmente, la presión de selección ejercida (Cerdeira y Duke, 2006; Taberner Palou et al., 2007).

Herbicidas como los inhibidores de la acetolactato sintasa (ALS) e inhibidores de la acetil coenzima-A carboxilasa (ACCasa) son capaces de seleccionar biotipos resistentes en 1-5 ciclos de selección (Mallory Smith et al., 1990). Esto se debe, principalmente, a la elevada especificidad del sitio activo, a la alta frecuencia de mutación del gen nuclear que codifica la enzima $\left(10^{-6}\right.$ para los herbicidas ALS) y a la posibilidad de que distintas mutaciones semidominantes alteren el sitio de acoplamiento del herbicida en la enzima y le confieran resistencia a la maleza (Delye et al., 2009; Tranel y Wright, 2002; Gressel, 2002).

Actualmente, los casos de resistencia a inhibidores de la ALS superan el centenar y abarcan 34 países. América Latina es uno de los grupos con mayor cantidad de malezas resistentes, con 16 especies confirmadas. Por su parte, el grupo de los inhibidores de la ACCasa, con 36 casos de resistencia, es otro de los de mayor contribución tanto para los indices mundiales como los de Latinoamérica, donde se registraron 16 casos particularmente en malezas gramíneas (Heap, 2010).

Aunque de menor dimensión que los dos casos anteriores, la resistencia al glifosato, inhibidor de la enzima enolpiruvyl-shikimato3-fosfato sintetasa (EPSPS), con 16 malezas resistentes, toma una relevancia especial debido a que su uso se ha generalizado a nivel mundial y particularmente en Argentina en virtud de la gran adopción del sistema Siembra Directa-Soja RR y a su creciente uso en barbechos químicos (Trigo y Cap, 2006).

Diversas especies de malezas que desarrollaron resistencia al glifosato fueron reportadas simultáneamente por presentar resistencia a otros principios activos (resistencia múltiple a herbicidas). Así, por ejemplo, malezas como Amaranthus rudis, Conyza spp., Eleusine indica, Euphorbia heterophylla, y Lolium rigidum presentan resistencia también a herbicidas como los inhibidores de la ACCasa o de la ALS (Yu et al., 2007; Heap, 2010).

En 2007, se reportó en Argentina resistencia a glifosato, en la provincia de Buenos Aires (Partido de Coronel Dorrego), en plantas de Lolium multiflorum (Diez de Ulzurrun et al., 2008; Vigna et al., 2008). Se sabe que dichas poblaciones fueron expuestas desde el año 1999 hasta el 2005 a 3-5 aplicaciones anuales de 400-500 g e.a. ha ${ }^{-1}$ de glifosato. A partir del año 2006, se registraron fallas en el control de tales poblaciones.

En el sudeste de la provincia de Buenos Aires, se registraron fallas de control a campo en poblaciones de Lolium multiflorum expuestas a distintos herbicidas de las familias de los inhibidores de ALS y ACCasa y al glifosato. Por ello, se planteó como objetivo caracterizar el nivel de resistencia a determinados herbicidas inhibidores de la ALS, de la ACCasa y al glifosato en una población supuestamente resistente del sudeste bonaerense.

\section{MATERIALES Y MÉTODOS}

Para confirmar y determinar el nivel de resistencia a herbicidas con diferentes modos de acción, se realizaron experimentos en cajas de Petri. Los ensayos fueron conducidos en cámara de crecimiento en el laboratorio de Terapéutica Vegetal de la Unidad Integrada Balcarce (EEA INTA Balcarce - Fac Cs. Agrarias UNMdP).

Se examinó una población supuestamente resistente de L. multiflorum del Partido de 
Loberia (Provincia de Buenos Aires, Argentina). Se colectaron las semillas de los grupos de plantas que sobrevivieron a las aplicaciones de campo con herbicidas. Dicha población fue denominada LmR y tenía una historia de, por lo menos, 5 o más años en siembra directa y con aplicaciones de glifosato y de graminicidas en cultivo y barbechos de trigo y cebada. Se utilizó como testigo susceptible una población cultivada de raigrás (Progrow, KWS Argentina).

Se colocaron 15 semillas en cada caja de Petri, sobre un papel de filtro embebido con $5 \mathrm{~mL}$ de alícuotas de distintas concentraciones de glifosato $(0 ; 12,5 ; 25 ; 50 ; 100 ; 200$; $400 \mathrm{mg}$ e.a. $\mathrm{L}^{-1}$ ), pyroxsulam, iodosulfuron, pinoxaden y clethodim $(0,001 ; 0,01 ; 0,1 ; 1 ; 10$; $100 ; 1.000$ micromolar $(\mu \mathrm{M}))$. Se realizaron tres repeticiones para cada una de las dosis. Dicho ensayo se llevó a cabo en una cámara de crecimiento con un régimen de 16 horas de luz y 8 horas de oscuridad y con una alternancia térmica de $15-20{ }^{\circ} \mathrm{C}$.

A los siete días después de la siembra, se determinó la longitud de la parte aérea (suma del largo de coleoptile y primera hoja en los casos en que ella estaba presente).

La longitud de coleoptile se expresó como porcentaje del control no tratado para estandarizar la comparación. Las curvas de dosisrespuesta para los bioensayos en caja de Petri fueron obtenidas por medio de un modelo de regresión no lineal usando la ecuación loglogística (Streibig, 1988; Streibig et al., 1993, 1995):

$$
\mathrm{Y}=\mathrm{C}+\left((\mathrm{D}-\mathrm{C}) /\left(1+\left(\mathrm{x} / \mathrm{GR}_{50}\right)^{\mathrm{b}}\right)\right.
$$

en que Y representa la longitud de coleoptile (como porcentaje del control no tratado) de plantas expuestas a dosis de los diferentes herbicidas (x), C es la respuesta media a una dosis de herbicida muy alta (limite inferior), D es la respuesta media cuando la dosis de herbicida tiende a cero (limite superior), b es la pendiente de la línea cuando hay una reducción del $50 \%$ del crecimiento $\left(\mathrm{GR}_{50}\right)$, y $\mathrm{GR}_{50}$ es la dosis de herbicida requerida para reducir el crecimiento en un 50\%. Los parámetros de regresión de los biotipos susceptibles y resistentes al glifosato fueron obtenidos usando Sigma Plot (versión 8.0, SPSS Inc, USA). El indice de resistencia (IR) se determinó mediante el cálculo de la relación entre la $\mathrm{GR}_{50}$ del biotipo resistente y la $\mathrm{GR}_{50}$ del biotipo susceptible.

\section{RESULTADOS Y DISCUSIÓN}

\section{Sensibilidad al glifosato}

La longitud de la parte aérea disminuyó para las poblaciones evaluadas expuestas a dosis crecientes de glifosato (Figura 1). E1 modelo no lineal propuesto proporcionó un buen ajuste a la relación entre la longitud de coleoptile (\% control no tratado) y las dosis de glifosato (Tabla 1). Se observó una menor sensibilidad al herbicida en la población $\mathrm{LmR}$ que en la población susceptible probada. La GR $\mathrm{Go}_{50}$ fue de $13,86( \pm 1,66)$ y 46,72 $( \pm 7,47)$ mg e.a. $\mathrm{L}^{-1}$ de glifosato en las poblaciones Progrow y LmR respectivamente.

El IR presentó un valor de 3,37 y confirmó la resistencia del biotipo $\mathrm{LmR}$ al herbicida glifosato. Dicho nivel de resistencia se encuentra en el rango de valores citados en la bibliografia para el herbicida glifosato, ya que, en general, la resistencia es del orden de 3-7 (LorraineColwill et al., 2002; Perez y Kogan, 2002; Wakelin et al., 2004; Perez Jones et al., 2007; Nandula et al., 2008; Preston y Wakelin, 2008; Vigna et al., 2008), con algunas excepciones en las cuales el IR superó el valor de 10 (Wakelin et al., 2004; Yu et al., 2007; Preston y Wakelin, 2008).

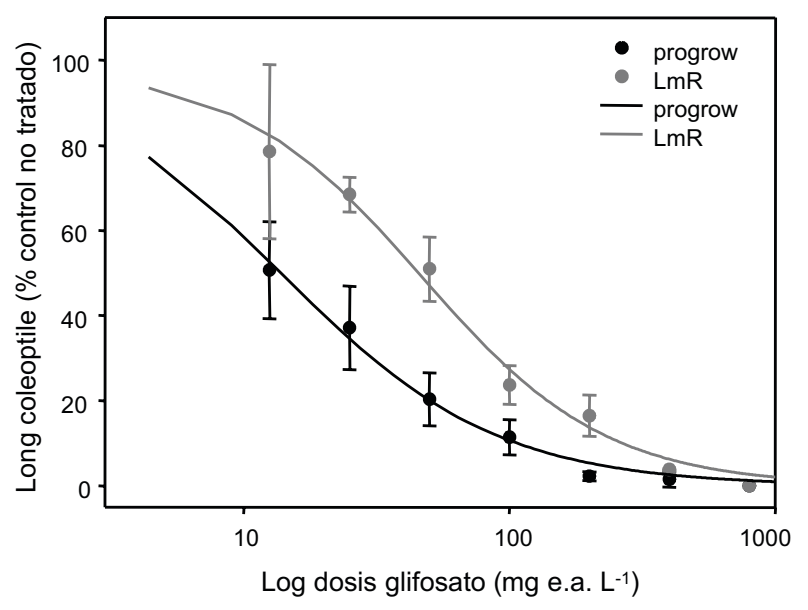

Figura 1 - Longitud del coleoptile de biotipos de Lolium multiflorum expuestos a dosis crecientes de glifosato. Los símbolos y líneas representan los valores reales y predichos respectivamente. Las barras verticales representan el error estándar de la media. 
Tabla 1 - Parámetros de regresión del modelo no lineal estimados y error estándar (SE) para los bioensayos en cajas con distintos herbicidas. El modelo ajustado corresponde a la longitud del coleoptile $(\%$ control no tratado $)=\mathrm{C}+[(\mathrm{D}-\mathrm{C}) / 1+(\mathrm{x} / \mathrm{LD} 50) \mathrm{b})]$

\begin{tabular}{|c|c|c|c|c|c|c|c|}
\hline Herbicida & Población & $\mathrm{D}( \pm \mathrm{SE})$ & $\mathrm{C}( \pm \mathrm{SE})$ & $\mathrm{b}( \pm \mathrm{SE})$ & $\begin{array}{c}\mathrm{GR}_{50}( \pm \mathrm{SE}) \\
\left(\mathrm{mg} \text { a.e. } \mathrm{L}^{-1}\right)\end{array}$ & $\mathrm{R}_{\mathrm{aj}}^{2}$ & IR \\
\hline \multirow{2}{*}{ Glifosato } & $\mathrm{LmR}$ & $98.4(4.50)$ & $0(5.13)$ & $1.25(0.22)$ & $46.700(7.470)$ & 0.95 & \multirow{2}{*}{3.37} \\
\hline & Progrow & $99.8(3.26)$ & $0(3.02)$ & $1.07(0.18)$ & $13.8600(1.660)$ & 0.97 & \\
\hline \multirow{2}{*}{ Pyroxsulam } & $\mathrm{LmR}$ & $97.0(3.87)$ & $0(3.60)$ & $0.68(0.11)$ & $0.5220(0.150)$ & 0.97 & \multirow{2}{*}{26.10} \\
\hline & Progrow & $105.0(8.00)$ & $0(8.00)$ & $0.19(0.07)$ & $0.0200\left(1.10^{-4}\right)$ & 0.97 & \\
\hline \multirow{2}{*}{ Iodosulfuron } & $\mathrm{LmR}$ & $98.0(2.95)$ & $0(2.00)$ & $0.71(0.02)$ & $0.1100(0.020)$ & 0.98 & \multirow{2}{*}{2.20} \\
\hline & Progrow & $101.0(2.97)$ & $0(3.95)$ & $0.28(0.05)$ & $0.0500(0.024)$ & 0.98 & \\
\hline \multirow{2}{*}{ Clethodim } & $\mathrm{LmR}$ & $85.4(3.50)$ & $0(5.80)$ & $1.73(0.29)$ & $0.1372(0.040)$ & 0.93 & \multirow{2}{*}{3.43} \\
\hline & Progrow & $94.0(2.22)$ & $0(2.29)$ & $1.32(0.17)$ & $0.0400(0.070)$ & 0.98 & \\
\hline \multirow{2}{*}{ Pinoxaden } & $\mathrm{LmR}$ & $91.7(2.80)$ & $0(4.85)$ & $2.35(0.23)$ & $0.1660(0.350)$ & 0.96 & \multirow{2}{*}{1.03} \\
\hline & Progrow & $97.8(0.80)$ & $0(1.50)$ & $5.16(0.92)$ & $0.1600(0.050)$ & 0.99 & \\
\hline
\end{tabular}

\section{Inhibidores de la ALS}

\section{Sensibilidad a pyroxsulam}

La longitud de la parte aérea disminuyó en las poblaciones evaluadas expuestas a concentraciones crecientes de pyroxsulam (Figura 2). La GR $\mathrm{GR}_{50}$ de la población $\mathrm{LmR}$ fue de $0,522 \mu \mathrm{M}$, mientras que, para el cultivo comercial, fue de $0,02 \mu \mathrm{M}$. Al calcular 1a relación de la $\mathrm{GR}_{50}$ del biotipo $\mathrm{LmR}$ y la del Progrow, obtuvimos un IR de 26,1, lo que indica un alto nivel de resistencia de dicha población al herbicida probado, perteneciente al grupo de inhibidores de la ALS.

\section{Sensibilidad a iodosulfuron}

La longitud de la parte aérea disminuyó para las poblaciones evaluadas expuestas a concentraciones crecientes de iodosulfuron (Figura 3). La GR $\mathrm{GR}_{50}$ de la población $\mathrm{LmR}$ fue de $0,11 \mu \mathrm{M}$, mientras que, para Progrow, fue de $0,05 \mu \mathrm{M}$. Al calcular la relación de la $\mathrm{GR}_{50}$ del biotipo LmR y la del Progrow, obtuvimos un IR de 2,2 , que indica la resistencia de la población LmR al herbicida iodosulfuron perteneciente también al grupo de los inhibidores de la ALS.

Espinoza et al. (2005) citan que el primer biotipo de L. multiflorum muestra resistencia múltiple a herbicidas el cual presenta un IR de 4 al glifosato y 4,6 al iodosulfurom. Espinoza et al. (2008) estudiaron la resistencia al iodosulfuron en biotipos resistentes al glifosato y verificaron que era de 2,8-6 en dichos biotipos con resistencia múltiple, que es similar a la del biotipo analizado en el presente estudio.

\section{Inhibidores de la ACCasa}

\section{Clethodim}

La longitud de la parte aérea disminuyó para las poblaciones evaluadas expuestas a dosis crecientes de clethodim (Figura 4). La longitud del coleoptile fue mayor para el biotipo Progrow para dosis de 0,001 y $0,01 \mu \mathrm{M}$, mientras que, con dosis superiores a $0,1 \mu \mathrm{M}$, la sensibilidad del Progrow fue mayor que la del biotipo $\mathrm{LmR}$. La $\mathrm{GR}_{50}$ de la población $\mathrm{LmR}$ fue de $0,1372 \mu \mathrm{M}$, mientras que para el Progrow fue de $0,04 \mu \mathrm{M}$. Al calcular la relación de la $\mathrm{GR}_{50}$ del biotipo LmR y la del Progrow, obtuvimos un IR de 3,43, que confirma la resistencia de la población $\mathrm{LmR}$ al herbicida clethodim perteneciente al grupo de los inhibidores de la ACCasa.

Espinoza et al. (2005) citan biotipos de L. multiflorum con resistencia a clethodim cuyos IR son de 7 a 11 veces mayores que los de los biotipos sensibles. Tales biotipos también mostraron resistencia cruzada con otros herbicidas inhibidores de la ALS.

En la especie L. rigidum, también se registraron biotipos con resistencia múltiple a herbicidas como glifosato, paraquat e inhibidores de la ACCasa (diclofop, haloxifop, fluazifop, propaquizafop, setoxidim y tralkoxidim) (Yu et al., 2007). 


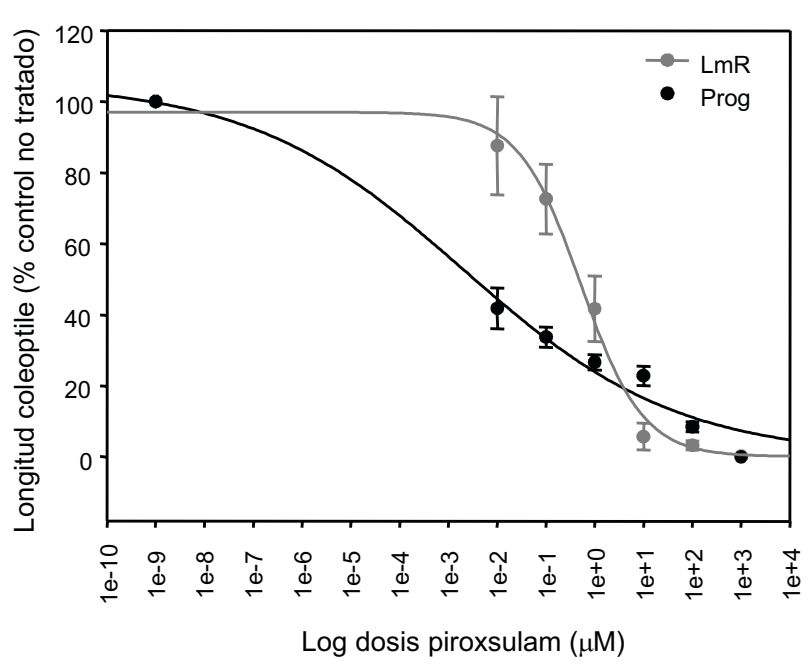

Figura 2 - Longitud del coleoptile de biotipos de Lolium multiflorum expuestos a dosis crecientes de piroxulam. Los símbolos y líneas representan los valores reales y predichos respectivamente. Las barras verticales representan el error estándar de la media.

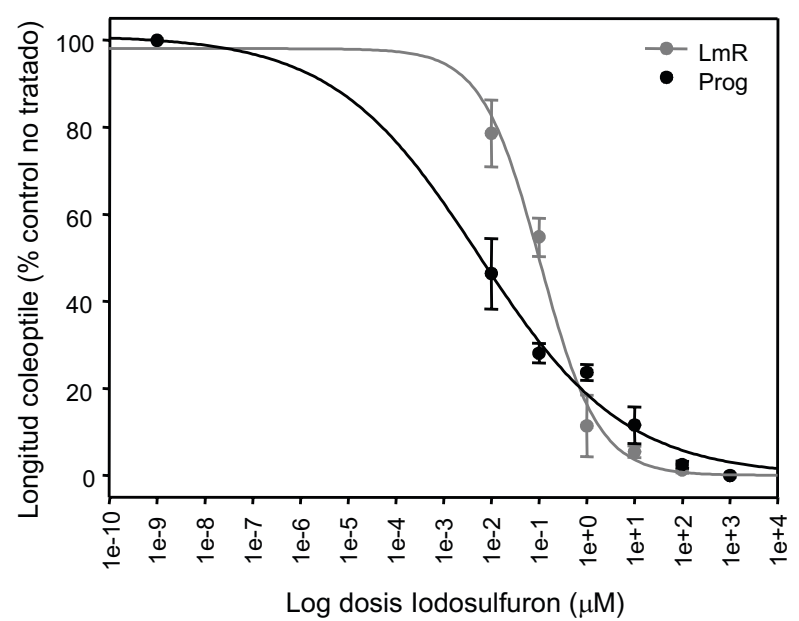

Figura 3 - Longitud del coleoptile de biotipos de Lolium multiflorum expuestos a dosis crecientes de iodosulfuron. Los símbolos y líneas representan los valores reales y predichos respectivamente. Las barras verticales representan el error estándar de la media.

\section{Pinoxaden}

La longitud de la parte aérea disminuyó para las poblaciones evaluadas expuestas a dosis crecientes de pinoxaden (Figura 5). La longitud del coleoptile fue similar para ambos biotipos en todas las dosis evaluadas. La $\mathrm{GR}_{50}$ de la población $\mathrm{LmR}$ fue de $0,166 \mu \mathrm{M}$, mientras que para Progrow fue de 0,16 $\mu \mathrm{M}$. Al calcular

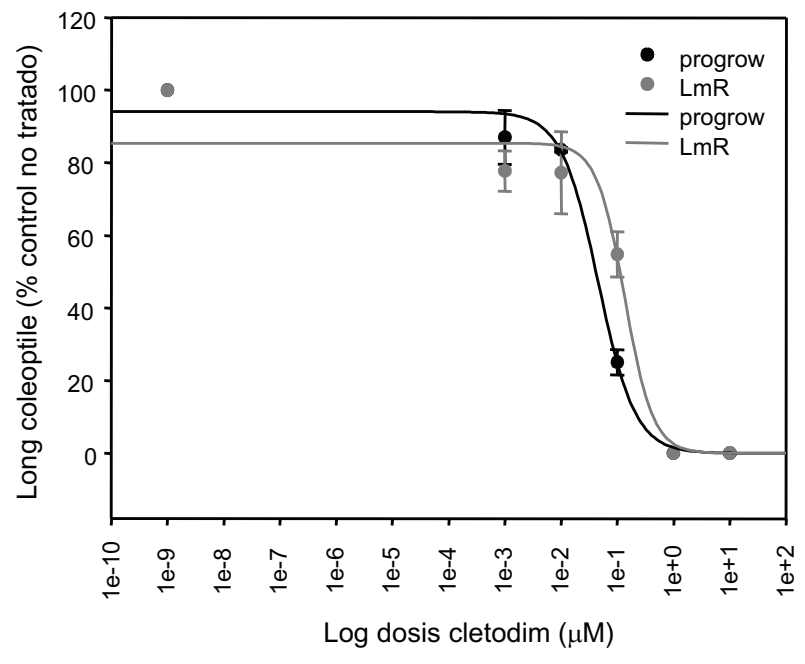

Figura 4 - Longitud del coleoptile de biotipos de Lolium multiflorum expuestos a dosis crecientes de clethodim. Los símbolos y líneas representan los valores reales y predichos respectivamente. Las barras verticales representan el error estándar de la media.

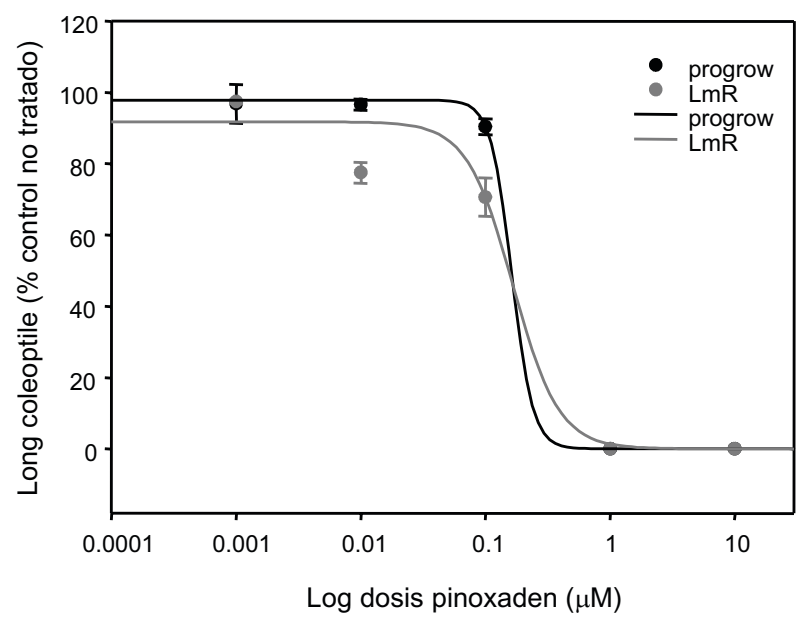

Figura 5 - Longitud del coleoptile de biotipos de Lolium multiflorum expuestos a dosis crecientes de pinoxadem. Los símbolos y líneas representan los valores reales y predichos respectivamente. Las barras verticales representan el error estándar de la media.

la relación de la $\mathrm{GR}_{50}$ del biotipo $\mathrm{LmR}$ y la del Progrow, obtuvimos un IR de 1,03, que indica que no hay resistencia de la población $\mathrm{LmR}$ a pinoxaden.

El biotipo LmR presentó resistencia múltiple a herbicidas con tres modos de acción diferentes, inhibidores de EPSPS, ALS y ACCasa. Dicho biotipo presentó un comportamiento 
similar a los biotipos chilenos descriptos por Espinoza et al. (2005).

Si bien se probaron varios principios activos con diferentes modos de acción, no se comprobó que todos ellos presentan resistencia, por lo cual se deberian realizar ensayos de campo para poder determinar fehacientemente la supuesta resistencia y evaluar herbicidas que sean efectivos en dosis adecuadas para establecer alternativas de control.

\section{LITERATURE CITED}

CERDEIRA, A. L.; DUKE, S. O. The current status and environmental impacts of glyphosate-resistant crops: a review. J. Environ. Qual., v. 35, n. 5, p. 1633-1658, 2006.

DÉLYE, C. et al. Variation in the gene encoding acetolactatesynthase in lolium species and proactive detection of mutant, herbicide-resistant alleles. Weed Res., v. 49, n. 3, p. 326-336, 2009.

DIEZ DE ULZURRUN, P. et al. Lolium multiflorum resistente a glifosato en la Provincia de Buenos Aires Presentación del proyecto de estudio. In: CONGRESSO BRASILEIRO DA CIENCIA DAS PLANTAS DANINHAS, 26; CONGRESO DE LAASOCIACIÓN

LATINOAMERICANA DE MALEZAS, 18., 2008, Ouro

Preto. Anales... Ouro Preto: 2008. 6 p

ESPINOZA, N. et al. Ballica (Lolium multiflorum Lam.) con resistencia a glifosato, glifosato-trimesium, iodosulfuron y flucarbazone sódico. In: CONGRESO DE LAASOCIACIÓN LATINOAMERICANA DE MALEZAS, 27.; CONGRESO IBEROAMERICANO DE CIENCIA DE LAS MALEZAS, 1., 2005, Varadero, Matanzas. Anales... Varadero, Matanzas: 2005. p. 324

ESPINOZA, N. et al. Resistencia múltiple a glifosato, ACCasa y ALS en biotipos de Lolium chilenos. In: CONGRESO LATINOAMERICANO DE MALEZAS, 18., 2008, Ouro Preto; CONGRESO BRASILEIRO DA CIENCIA DAS PLANTAS DANINHAS, 26., 2008, Ouro Preto. Anales... Ouro Preto, 2008. 12 p.

GRESSEL, J.; SEGEL, L. A. The paucity of genetic adaptive resistance of plants to herbicides: possible biological reasons and implications. J. Theor. Biol., v. 75, n. 3, p. 349-371, 1978.

GRESSEL, J. Molecular biology of weed control. London/ New York: Taylor \& Francis. 2002. 504 p.

HEAP, I. M. International survey of herbicide resistant weeds. 2010. Disponible en: $<\mathrm{http}: / / \mathrm{www}$.weedscience.com $>$ Consultado em: 13 abr. 2010
LORRAINE-COLWILL, D. F. et al. Investigation into the mechanism of glyphosate resistance in Lolium rigidum Pestic. Biochem. Physiol., v. 74, n. 2, p. 62-72, 2002

MALLORY-SMITH, C. A. P.; HENDRICKSON, P.; MUELLER-WARRANT, G. W. Identification of herbicide resistant prickly lettuce (Lactuca serriola). Weed Technol. v. 4 , p. $163-168,1990$

NANDULA, V. K. et al. Glyphosate tolerance mechanism in Italian ryegrass (Lolium multiflorum) from Mississippi. Weed Sci., v. 56, n. 3, p. 344-49, 2008.

PÉREZ, A.; KOGAN, M. Glyphosate-resistant Lolium multiflorum in Chilean orchards. Weed Res., v. 43, n. 1, p. $12-19,2002$

PEREZ JONES, A. et al. Investigating the mechanism of glyphosate resistance in lolium multiflorum. Planta, v. 226, n. 2, p. 395-404, 2007.

POWLES, S. B.; YU, Q. Evolution in action: plants resistant to herbicides. Ann. Rev. Plant Biol., v. 61, n. 1, p. 317-347, 2010

SEEFELDT, S. et al. Log-logistic analysis of herbicide rateresponse relationships. Weed Technol., v. 9, n. 2, p. 218227, 1995.

STREIBIG, J. C. Herbicide bioassay. Weed Res., v. 28, n. 6, p. $479-484,1988$

STREIBIG, J. C. et al. Dose response curves and statistical models. In: STREIBIG, J. C.; KUDSK, P. (Eds.). Herbicide bioassays. Boca Raton: CRC Press, 1993. p. 30-55.

TABERNER PALOU, A. et al. Manejo de poblaciones de malezas resistentes a herbicidas: 100 preguntas sobre resistencias. 2007. 78 p. Disponible en: $<$ http://www.fao.org/ ag/AGP/AGPP/IPM/Weeds/Download/manejo.pdf>. Consultado en: 13 de abril de 2010.

TRANEL, P. J.; WRIGHT, T. R. Resistance of weeds to ALS-inhibiting herbicides: What have we learned? Weed Sci., v. 50, n. 6, p. $700-712,2002$

TRIGO, E. J.; CAP. E. Diez años de cultivos genéticamente modificados en la agricultura Argentina. ARGENBIO. En línea, 2006. Disponible en: <http://www.inta.gov.ar/ies/docs/ otrosdoc/Diez_anos_cultivos_GM_Argentina.pdf>. Consultado en: 20 maio 2009

VIGNA, M. R. et al. Estudios de curvas dosis-respuesta de poblaciones de Lolium multiflorum a glifosato en el SO de Buenos Aires, Argentina. In: CONGRESSO BRASILEIRO DA CIÊNCIA DAS PLANTAS DANINHAS, 26; CONGRESO DE LAASOCIACIÓN LATINOAMERICANA DE MALEZAS, 18., 2008, Ouro Preto. Anales... Ouro Preto, 2008. 13 p. 
PRESTON, C.; WAKELIN, A. M. Resistance to glyphosate from altered herbicide translocation patterns. Pest Manag. Sci., v. 64, n. 4, p. 372-376, 2008

WAKELIN, A. M. et al. Glyphosate resistance in four different populations of Lolium rigidum is associated with reduced translocation of glyphosate to meristematic zones. Weed Res., v. 44, n. 6, p. 453-459, 2004
WSSA. Herbicide resistance and herbicide tolerance defined. Weed Technol., v. 12, n. 4, p. 789, 1998.

YU, Q. et al. Glyphosate, paraquat and ACCase multiple herbicide resistance evolved in a Lolium rigidum biotype. Planta, v. 225, n. 2, p. 499-513, 2007.

YUAN, J. S. et al. Non-target site herbicide resistance: a family business. Trends Plant Sci., v. 12, n. 1, p. 6-13, 2006 